\title{
Circulating insulin-like growth factors and Alzheimer disease
}

\section{A mendelian randomization study}

Dylan M. Williams, PhD, Ida K. Karlsson, MSc, Nancy L. Pedersen, PhD, and Sara Hägg, PhD

Neurology ${ }^{\circledR}$ 2018;90:e291-e297. doi:10.1212/WNL.0000000000004854

\section{Abstract}

\section{Objective}

To examine whether genetically predicted variation in circulating insulin-like growth factor 1 (IGF1) or its binding protein, IGFBP3, are associated with risk of Alzheimer disease (AD), using a mendelian randomization study design.

\section{Methods}

We first examined disease risk by genotypes of 9 insulin-like growth factor (IGF)-related single nucleotide polymorphisms (SNPs) using published summary genome-wide association statistics from the International Genomics of Alzheimer's Project (IGAP; $n=17,008$ cases; 37,154 controls). We then assessed whether any SNP-disease results replicated in an independent sample derived from the Swedish Twin Registry ( $\mathrm{n}=984$ cases; 10,304 controls).

\section{Results}

Meta-analyses of SNP-AD results did not suggest that variation in IGF1, IGFBP3, or the molar ratio of these affect $A D$ risk. Only one $S N P$ appeared to affect $A D$ risk in IGAP data. This variant is located in the gene FOXO3, implicated in human longevity. In a meta-analysis of both IGAP and secondary data, the odds ratio of $\mathrm{AD}$ per FOXO3 risk allele was 1.04 (95\% confidence interval 1.01-1.08; $p=0.008$ ).

\section{Conclusions}

These findings suggest that circulating IGF1 and IGFBP3 are not important determinants of $\mathrm{AD}$ risk. FOXO3 function may influence $\mathrm{AD}$ development via pathways that are independent of IGF signaling (i.e., pleiotropic actions).

\author{
Correspondence \\ Dr. Williams \\ Dylan.williams@ki.se
}




\section{Glossary}

AD = Alzheimer disease; $\mathbf{C I}$ = confidence interval; GWAS = genome-wide association study; IGAP = International Genomics of Alzheimer's Project; IGF = insulin-like growth factor; IQR = interquartile range; IVW = inverse-variance weighting; $\mathbf{M R}=$ mendelian randomization; $\mathbf{O R}=$ odds ratio; SNP = single nucleotide polymorphism; STR = Swedish Twin Registry.

The insulin-like growth factor (IGF) axis may have a role in cognitive decline and the etiology of Alzheimer disease (AD). ${ }^{1}$ Activity of the axis decreases markedly with age throughout adulthood, with circulating growth hormone/ IGF1 very low in those aged over 60 years. ${ }^{2}$ IGF1 purportedly has neuroprotective effects in adult animals, promoting neuronal survival and reducing tau phosphorylation., ${ }^{3,4}$ Consistent with experimental findings, epidemiologic studies have found that individuals with $\mathrm{AD}$, all-cause dementia, or cognitive decline have lower circulating IGF1 and its main binding protein (IGFBP3) than cognitively intact individuals, $^{5-9}$ although some findings have been contradictory. ${ }^{10-12}$ Higher circulating IGF2 exposure could also have a role in delaying cognitive decline. ${ }^{10}$

Based on these past findings, circulating IGF1 has been proposed by some as a modifiable target for $\mathrm{AD}$ treatment or prevention to test in trials. ${ }^{6}$ It therefore remains to be clarified as to whether IGF concentrations in circulation are a causal factor in $\mathrm{AD}$ etiology. To investigate this further using a novel approach, we tested the hypothesis that risk of developing $\mathrm{AD}$ would be lower in individuals with genetically predicted, long-term increases in exposure to circulating IGF1 and IGFBP3.

\section{Methods}

\section{Study design}

We conducted a mendelian randomization (MR) analysis (figure 1$)^{13}$ using a 2 -sample design: first identifying genetic variants that affect circulating IGF concentrations from published genetic datasets, and then assessing genotype-outcome associations for each identified genetic variant in secondary $\mathrm{AD}$ case-control datasets. ${ }^{14}$

\section{Genetic variant selection}

To identify variants as genetic instruments for IGF exposure, we used information from the largest genome-wide association study (GWAS) of circulating IGF1 and IGFBP3 to date. $^{15}$ This identified single nucleotide polymorphisms (SNPs) at 10 independent loci below the threshold for genome-wide significance $\left(p\right.$ values $\left.<5 \times 10^{-8}\right)$. Analyses of IGF 1 combined data on up to 30,884 individuals $(53.3 \%$ female) from 21 cohort studies. Analyses of IGFBP3 combined data on up to 18,995 individuals ( $57.6 \%$ female) from 13 cohorts. All participants were of European ancestry. Mean ages within cohorts ranged from 18.9 to 76 years. The published GWAS contains full information on the consortium's studies, participants, genotyping, and IGF assays. ${ }^{15}$
Variants at 7 of the loci determine IGF1, and 4 determine IGFBP3; hits at 2 loci affect both traits. Most of the loci have known or plausible biological links to IGF axis activity. ${ }^{15,16} \mathrm{~A}$ 10th locus had been found to determine both traits in opposite directions in a bivariate analysis. Hence, the top SNP at this locus was omitted from the main analysis due to potentially conflicting pleiotropic effects on exposures of interest, leaving 9 variants to use, but the additional variant was included in a sensitivity analysis (described below).

\section{Primary AD case-control sample}

Our primary sample for examining genotype-outcome associations consisted of 17,008 late-onset $\mathrm{AD}$ cases and 37,154 controls of European ancestry included in the stage 1 GWAS meta-analysis conducted by the International Genomics of Alzheimer's Project (IGAP). ${ }^{17}$ IGAP has published summary statistics of genotype$\mathrm{AD}$ associations for 7,055,881 SNPs online (web.pasteur-lille.fr/ en/recherche/u744/igap/igap_download.php). Cases within the

Figure 1 Directed acyclic graph illustrates the mendelian randomization approach

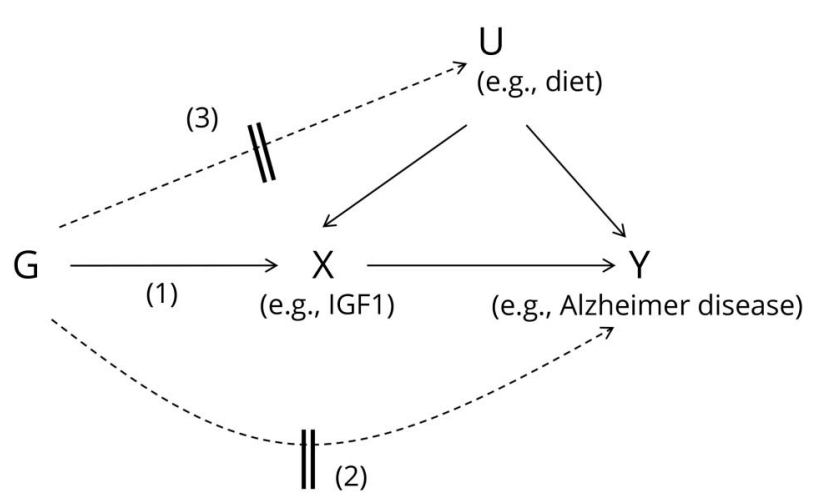

Observational studies may have established an association between an exposure $(X)$, such as variation in insulin-like growth factors (IGFs), and outcome $(Y)$, such as risk of Alzheimer disease. These studies will be biased from confounding $(U)$ of the $X-Y$ association that is unmeasured/uncontrolled by statistical models, and possibly other sources of bias such as reverse causation. Mendelian randomization can help to assess whether the exposure is causally related to outcome by using a genetic variant (G) (or several in combination) as an instrumental variable for an exposure. This assumes that the genotypes are robust determinants of the exposure (pathway 1). Due to the independent assortment of alleles for variants between parents and offspring at conception, genotypes that determine the exposure should not also determine confounding factors, nor should disease status modify the genotype (reverse causation). ${ }^{13}$ Therefore, G-Y associations should help to infer a causal relationship of $X$ with $Y$ if instrumental variable assumptions hold. There are potential violations to the framework that can induce direct association of genotypes with outcome independently of the exposure and confounders (pathway 2), or indirectly via confounders (pathway 3). For example, these could arise from horizontal pleiotropy (variants having multiple effects that are independent of exposure determination), linkage disequilibrium between the instrumenting variants and others that affect other traits, or population stratification leading to clustering of variant genotypes and confounding traits. 
consortium's cohorts had mean ages at onset ranging from 68.5 to 82.3 years, of which approximately $60 \%$ were women. More details on the stage 1 studies, participants, genotype data, $\mathrm{AD}$ diagnostic criteria, and statistical models are described in the published GWAS. ${ }^{17}$

\section{Replication AD case-control sample}

To test for replication of any SNP-AD findings of note, we derived secondary samples of $\mathrm{AD}$ cases and controls with genome-wide data available from 4 substudies of individuals in the Swedish Twin Registry (STR). ${ }^{18-21}$ Appendix e-1 (links. lww.com/WNL/A60) contains a detailed description of the sample. Its derivation is depicted in figure e-1 (links.lww.com/ $\mathrm{WNL} / \mathrm{A} 58)$, and descriptive characteristics are shown in table e-1 (links.lww.com/WNL/A59). In total, the samples consisted of 984 cases and 10,304 controls.

\section{Sample overlap}

We attempted to quantify the degree of overlap between participants included in the GWAS consortia for IGFs and AD (table e-2, links.lww.com/WNL/A59), which could bias 2 -sample MR results if substantial. ${ }^{22}$ The precise degree of overlap could not be determined, but of 19 cohorts included in the main IGF GWAS, ${ }^{15} 4$ were also part of the IGAP consortium. Up to 10,657 of 30,884 participants (34.5\%) included in the GWAS of IGF1 may have been in the IGAP case-control sample (mainly as controls), and up to 8,228 of $18,995(43.3 \%)$ included in the GWAS of IGFBP3. True proportions were probably smaller. Risk of bias from sample overlap is therefore likely to be low.

\section{Statistical analysis}

We based our main analysis on genotype- $\mathrm{AD}$ data within $\mathrm{AD}$ case-control samples, using IGAP's logistic regression models with genotype as the independent variable, and age, sex, and principal components as covariates. These analyses do not estimate the magnitude of $\mathrm{AD}$ risk change per unit difference in the exposure, as other MR models do, ${ }^{14}$ but still help to infer causality and direction of any effects of exposures and outcomes and also benefit from less stringent model assumptions. ${ }^{23}$ For the 9 SNPs of relevance, we extracted $\beta$ coefficients, standard errors, and the coded effect alleles for logistic regression results of SNP-AD effects from the IGAP meta-analysis. We recoded 5 of the betas by subtracting log-odds values from zero, so that all $9 \mathrm{SNP}-\mathrm{AD}$ results were consistently expressed as odds ratios (ORs) and 95\% confidence intervals (CIs) according to IGFraising allele counts of the variants (table 1 for coding and annotation of SNP locations from the dbSNP database; ncbi. nlm.nih.gov/SNP/). We assumed additive effects of allele copies on IGFs. Individual SNP-AD results were combined in a fixed-effects meta-analysis using inverse-variance weighting (IVW) - the overall estimate offering more precision for evidence of causality of IGF effects on $\mathrm{AD}$ risk.

\section{Sensitivity analyses}

Circulating IGF binding proteins may have metabolic effects that are independent of their role in transporting the 2 main
IGF ligands. ${ }^{24}$ Thus, we conducted separate analyses to examine whether each genetically instrumented IGF peptide may affect $\mathrm{AD}$ risk, with a meta-analysis combining results for the set of 5 SNPs solely affecting total circulating IGF1, and a second meta-analysis combining results for 2 SNPs solely affecting IGFBP3. ${ }^{15}$

The molar ratio of IGF1 to IGFBP3 in circulation may be a better measure of the bioavailability of free IGF1 than separate measures of either component, ${ }^{25}$ and this ratio has been suggested to affect $\mathrm{AD}$ risk (where constituent measures have not) in some studies. ${ }^{9}$ Therefore, we conducted a third sensitivity analysis that coded variants according to genotypes expected to increase the ratio of IGF1 to IGFBP3. This metaanalyzed $5 \mathrm{SNP}-\mathrm{AD}$ results coding on alleles that raise IGF1 but do not affect IGFBP3 at genome-wide significance, alongside 2 SNPs coded on alleles that lower IGFBP3 but appear not to affect IGF1. In this analysis, we also included an additional variant (rs646776) that appears to have inverse effects on IGF1 and IGFBP3, coding on the allele leading to higher IGF1 and lower IGFBP3. ${ }^{15}$

\section{Estimating the magnitude of IGF-AD effects}

MR estimates of effect magnitudes using the ratio of coefficient method (or others) were precluded for the main analysis because the requisite $\beta$ coefficients and standard errors for the 9 SNP-IGF results were not reported in the published GWAS, nor available online (results were only available as weighted $z$ scores). ${ }^{15,26}$ However, using summary statistics published in another previous GWAS, ${ }^{16}$ a subset of 7 variants were applied for this purpose in an additional analysis of IGF1 (with 3 variants) and IGFBP3 (with 4 variants). We used one set of study-level $\beta$ s and standard errors of SNP-IGF results from the Framingham Heart Study sample. SNP-IGF results were scaled consistently by SD increases in the peptides per copy of the coded alleles. We calculated SDs from the study interquartile ranges (IQRs) for the IGF peptides, assuming approximately normal distributions of the variables (in which an SD = IQR/ 1.35 in large samples). ${ }^{27}$ Wald estimators were calculated by dividing the SNP-AD result $\beta$ s by the SNP-IGF result $\beta$ s, and standard errors were derived by the delta method. ${ }^{14}$ We then combined Wald estimators for each SNP-AD result in fixedeffects meta-analyses with IVW, giving overall estimates of $\mathrm{AD}$ risk difference per $\mathrm{SD}$ increases in genetically predicted IGF1 and IGFBP3.

\section{Testing for violations of MR assumptions}

The instrumental variable assumptions made for our models can be violated in several ways (figure 1). ${ }^{13}$ Heterogeneity of SNP-AD results can help to identify variants that may be having pleiotropic effects, and so we reported heterogeneity test statistics from meta-analysis models (Cochran $Q$ and $\left.I^{2}\right) .{ }^{14}$ We considered the use of MR-Egger regression, as a further tool to address pleiotropy. ${ }^{28}$ However, this approach lacks effectiveness when using a small number of variants to instrument exposures, and has lower statistical power for 
Table 1 Descriptive information and statistics for the 9 single nucleotide polymorphisms (SNPS) determining insulin-like growth factor 1 (IGF1) or IGFBP3, and their associations with Alzheimer disease (AD) risk in the International Genomics of Alzheimer's Project (IGAP) dataset ( $n=17,008$ cases; 37,154 controls)

\begin{tabular}{|c|c|c|c|c|c|c|c|c|c|c|}
\hline SNP & Chr & $\begin{array}{l}\text { Nearest } \\
\text { gene }\end{array}$ & $\begin{array}{l}\text { SNP site/ } \\
\text { functional } \\
\text { relevance }\end{array}$ & $\begin{array}{l}\text { Determinant } \\
\text { of IGF } \\
\text { peptides }^{a}\end{array}$ & $\begin{array}{l}\text { Coded } \\
\text { allele }^{\text {b }}\end{array}$ & $\begin{array}{l}\text { Alternate } \\
\text { allele }\end{array}$ & $\begin{array}{l}\text { Coded } \\
\text { allele } \\
\text { frequencyc }\end{array}$ & $\begin{array}{l}\text { OR for AD per } \\
\text { coded allele }^{d}\end{array}$ & $95 \% \mathrm{Cl}$ & $\begin{array}{l}p \\
\text { Value }\end{array}$ \\
\hline rs700753 & 7 & TNS3 & $\begin{array}{l}\text { Intergenic/ } \\
\text { IncRNA }\end{array}$ & Both & G & $C$ & 0.65 & 1.01 & $0.98-1.04$ & 0.51 \\
\hline rs780093 & 2 & GCKR & Intronic & IGF1 & C & $\mathrm{T}$ & 0.59 & 1.00 & $0.97-1.04$ & 0.78 \\
\hline rs978458 & 12 & IGF1 & Intronic/ncT & IGF1 & T & C & 0.26 & 0.99 & $0.96-1.03$ & 0.63 \\
\hline rs2153960 & 6 & FOXO3 & Intronic & IGF1 & A & G & 0.69 & 1.04 & $1.01-1.08$ & 0.02 \\
\hline rs934073 & 2 & ASXL2 & $\begin{array}{l}\text { Intergenic/ } \\
\text { unknown }\end{array}$ & IGF1 & G & C & 0.29 & 1.01 & $0.98-1.05$ & 0.51 \\
\hline rs1065656 & 16 & NUBP2 & $\begin{array}{l}\text { 3'-UTR/ } \\
\text { missense }\end{array}$ & Both & G & C & 0.69 & 1.00 & $0.96-1.03$ & 0.87 \\
\hline rs509035 & 3 & GHSR & Intronic & IGF1 & A & G & 0.31 & 1.00 & $0.97-1.03$ & 0.90 \\
\hline rs11977526 & 7 & IGFBP3 & Intronic & IGFBP3 & A & G & 0.41 & 0.99 & $0.96-1.02$ & 0.59 \\
\hline rs4234798 & 4 & SORCS2 & Intronic & IGFBP3 & G & $\mathrm{T}$ & 0.61 & 1.00 & $0.97-1.03$ & 0.91 \\
\hline
\end{tabular}

Abbreviations: $\mathrm{Cl}$ = confidence interval; GWAS = genome-wide association study; IncRNA = variant lies in a long noncoding RNA; ncT = transcript variant in a noncoding RNA; OR = odds ratio; UTR = untranslated region at the end of a gene.

a Variants identified as determinants of IGF1 or IGFBP3 by association tests with $p$ values below the threshold for genome-wide significance in the cited GWAS of IGF1 and IGFBP3.

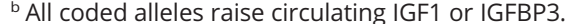

'Allele frequencies are those reported in the GWAS of IGF1 and IGFBP3; these were not presented in IGAP stage 1 summary statistics.

${ }^{\mathrm{d}} \mathrm{ORs}, 95 \% \mathrm{Cls}$, and $p$ values for AD per coded allele, assuming additive risk differences per allele copies (results plotted in figure 2 ).

overall effect inference than the IVW method. We did not therefore apply the additional method in this analysis.

\section{Power calculation}

To examine whether we had an effective sample size to undertake the MR analyses, we conducted a power calculation using a published calculator. ${ }^{29}$ This estimated the power for analyses to detect a minimum $\mathrm{OR}$ for $\mathrm{AD}$ risk per SD difference in IGFBP3 concentration. This used the study-level average $R^{2}$ statistic (6.5\%) for variance explained in IGFBP3 by the top 4 SNPs determining trait variation in one of the reported GWAS, ${ }^{16}$ along with the sample size $(\mathrm{n}=54,162)$ and proportion of cases $(0.314)$ in the stage 1 IGAP sample. The corresponding $R^{2}$ value was not reported for SNP-IGF1 results, so we did not power calculate for the analysis of IGF1.

\section{Replication analysis}

Statistical modeling details for the STR data are provided in appendix e-1 (links.lww.com/WNL/A60).

All analyses were conducted in Stata (version 14.2) and R (version 3.2.2), including the use of $\mathrm{R}$ package MR Base. ${ }^{30}$

\section{Standard protocol approvals, registrations, and patient consents}

Written informed consent was obtained from study participants in IGAP and the substudies of the STR or, for those with substantial cognitive impairment within IGAP, from a caregiver, legal guardian, or other proxy instead. IGAP study protocols were reviewed by the local or institutional ethics review boards of the consortium's studies. STR data use was approved by a regional ethics board in Stockholm (DNR 2015/1729-31/5).

\section{Results}

Table 1 shows statistics for the 9 SNPs used in the main analysis for causal inference. Results of the fixed-effects metaanalysis of the 9 genotype- $\mathrm{AD}$ effect estimates are shown in figure 2. The overall estimate indicated no effect of IGF1 or IGFBP3 variation on $\mathrm{AD}$ risk. No individual genotype appeared to noticeably affect $\mathrm{AD}$ risk, with one exception: rs2153960, which lies in the first intron of the FOXO3 gene. There was no evidence of heterogeneity between SNP-AD results, meaning that estimates consistently centered across the null. Separate analyses of SNPs solely affecting either IGF1 (figure e-2, links.lww.com/WNL/A58) or IGFBP3 (figure e-3) with $\mathrm{AD}$ risk also indicated overall null findings for both sets of variants. Similarly, $\mathrm{AD}$ risk did not appear to differ discernibly by differences in genetically instrumented molar ratio of IGF1 to IGFBP3 (figure e-4).

Figures e-5 and e-6 (links.lww.com/WNL/A58) show the estimates of the magnitude of effect of variation in IGF1 and 
Figure 2 Associations of insulin-like growth factor (IGF)-determining single nucleotide polymorphism (SNP) genotypes with Alzheimer disease (AD) risk, International Genomics of Alzheimer's Project data ( $n=17,008$ cases; 37,154 controls)

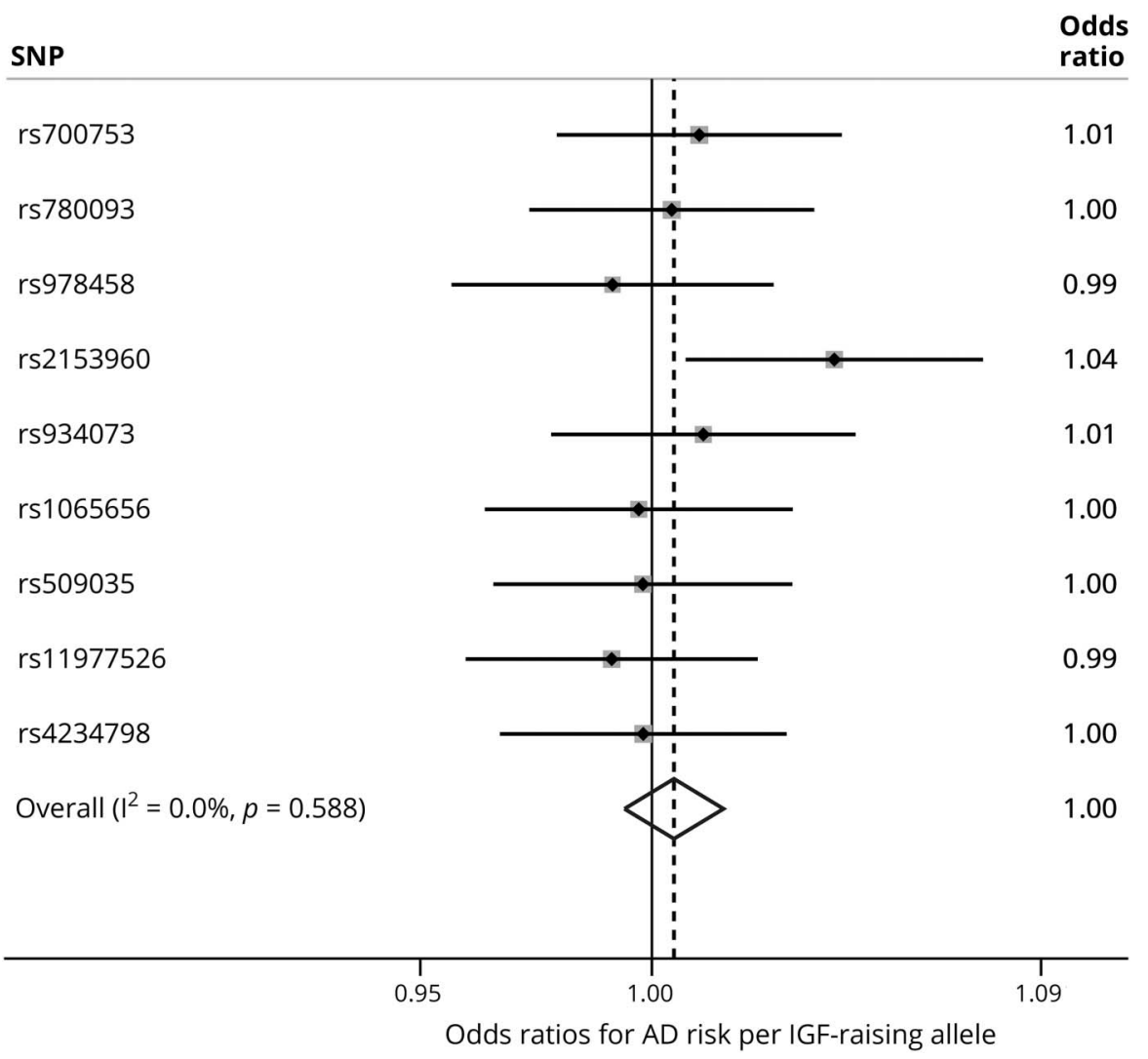

Odds

.01

.00

99

04

01

00 interval from fixed-effects meta-analysis is shown by the diamond's central position and lateral width, respectively, along with the test statistic of heterogeneity between individual estimates $\left(I^{2}\right)$. Gray boxes around point estimates indicate the weighting of results in the overall estimate.
IGFBP3 on AD risk (rather than just inferring causality and effect direction, as in the main analysis). An SD increase in circulating IGF1 would not appear to lead to lower $\mathrm{AD}$ risk. There was also no evidence for an effect of an SD increase in IGFBP3 on AD risk. Some heterogeneity was present in the 3 individual IGF1-AD effect estimates-likely driven by the disparity of the result for the FOXO3 variant rs2153960, compared to the 2 others. No heterogeneity was observed among the 4 IGFBP3-AD effect estimates.

The power calculation for our MR analysis indicated that the sample size should have been effective for identifying an OR of approximately 0.90 or lower per SD increase in IGFBP3 at $80 \%$ power, if higher IGFBP3 exposure would be expected to lessen $\mathrm{AD}$ risk (or, conversely, an $\mathrm{OR} \sim 1.12$ if expected to raise risk).

In the secondary analysis of SNP rs2153960 at the FOXO3 locus in relation to $\mathrm{AD}$ risk, the result was replicated in one sample of STR twins from the TwinGene cohort, but the point estimate was close to the null in the second subsample (figure e-7, links.lww.com/WNL/A58). CIs for both were wide and overlapped with the IGAP estimate-possibly indicating vibration of imprecise estimates around a small effect of the coded allele with $\mathrm{AD}$ risk. The overall meta-analysis result for IGAP and STR samples suggested slightly stronger evidence for effect than the IGAP result did alone.

\section{Discussion}

These findings do not support the hypothesis that long-term variation in circulating IGF1 or IGFBP3 cause differences in $\mathrm{AD}$ risk. Only 1 of 9 variants that instrumented circulating IGF peptides appeared to affect $\mathrm{AD}$ risk, and this may have arisen from pleiotropic effects-i.e., via the influence of the FOXO3 locus on alternate metabolic pathways, independent of its effects on the IGF axis.

The lack of effect of genetically predicted IGF1 and IGFBP3 on $\mathrm{AD}$ risk contrasts many of the conventional case-control studies that reported that disease risk was raised in those with lower circulating measures of IGF peptides. ${ }^{7,8,11,12,31,32}$ Given that measured circulating IGFs are highly responsive to concurrent influences of lifestyle factors that could be affected by disease status, such as recent physical activity, these studies may be particularly prone to bias from reverse causation. Two prospective studies have also investigated whether baseline circulating IGF peptides predict later incident $\mathrm{AD}$ risk. ${ }^{6,10}$ Among 3,582 individuals in the US Framingham Heart Study, those grouped in the lowest quartile of baseline IGF1 
measures had a higher incidence of $\mathrm{AD}$ than participants with higher quartiles (hazard ratio 1.52, 95\% CI 1.14-2.00). ${ }^{6}$ In contrast, a study of 745 British men did not find that baseline circulating IGF1, IGFBP3, IGF1:IGFBP3 molar ratio, or IGF2 predicted incident dementia risk (not limited to $A D$ ) after 17 years of follow-up, although only 40 dementia cases were recorded. ${ }^{10}$ Given the long prodromal period of $\mathrm{AD}$ development (perhaps several decades), reverse causation remains a potential source of bias for prospective studies, including for findings from the Framingham Heart Study samples (mean follow-ups 7 and 8.8 years). ${ }^{6}$ Moreover, residual confounding will have influenced all past observational findings. MR studies are not prone to these typical biases (but come with separate limitations). ${ }^{13}$ Hence, the lack of $\mathrm{AD}$ risk difference according to genetically predicted variations in IGF1, IGFBP3, and the molar ratio of these suggests that previous observational evidence implicating these circulating IGF peptides in $\mathrm{AD}$ etiology may have been misleading.

It is intriguing to observe a variant within the FOXO3 locus is linked to $\mathrm{AD}$ risk, considering consistent evidence linking FOXO3 to longevity in animal models and human samples. $^{33,34}$ Given the result's incongruence among 8 other null findings for IGF-determining variants, pleiotropic effects may link FOXO3 function with AD independently of FOXO3 effects on IGFs. In a GWAS of human longevity (living $\geq 90$ years vs dying sooner), another SNP in FOXO3, which is in high LD with rs2153960 ( $\left.r^{2} \sim 0.9\right)$, ranked highly. ${ }^{34}$ Taking these findings together, the A allele of rs 2153960 is linked with both higher risk of $\mathrm{AD}$ and a lower chance of reaching the age of 90 years. If variants in the first FOXO3 intron are truly related to $\mathrm{AD}$ via cis effects on $\mathrm{FOXO} 3$ production, there could be 2 explanations for this link. First, FOXO3 expression could affect $\mathrm{AD}$ development directly, perhaps via effects on apoptosis in neurons or genetic buffering of $A P O E$ or other loci. $^{35,36}$ Second, FOXO3 could affect AD risk indirectly via susceptibility to other diseases (i.e., competing risks of death) occurring earlier in the life course.

Strengths of the study include the use of very large $\mathrm{AD}$ case-control data, giving sufficient power to detect even small effects. Using multiple variants as instruments for IGF1 and IGFBP3 provided means to identify pleiotropy, which can bias single instrument results in either direction. There are also limitations to this evidence. The SNPs used in these analyses are not ideal instruments for single IGF peptides: several that affect IGF1 also appear to alter IGFBP3 concentrations and vice versa, and perhaps also influence circulating IGF2 (for which we did not find suitable variants to instrument specifically). ${ }^{37}$ Therefore, variants are not indicating $\mathrm{AD}$ risk differences attributable to one IGF molecule specifically, although sensitivity analyses using subsets of SNPs that were carefully assessed for magnitudes of effects on each peptide can help in this respect. Moreover, effects of variants on multiple IGF peptides may not invalidate their use as instruments for IGF axis activity as a whole. ${ }^{37}$ We did not address the potential for insulin to influence $\mathrm{AD}$ risk; a point of relevance, given that insulin binds to IGF receptors. However, a previous $\mathrm{MR}$ study found that $\mathrm{AD}$ risk did not differ by genetically predicted variation in insulin. ${ }^{38}$ Population stratification may be another source of bias for MR studies, ${ }^{13}$ but samples were of homogeneous ancestry, and principal components controlling for stratification were included in all analyses. Since the study samples were of white European ancestry, the results are most generalizable to populations of this ethnicity, and less so to others. Finally, MR generally assesses effects of lifelong variation in traits and does not reflect sensitive exposure windows. ${ }^{13}$ This may be important if developmental exposure is critical for later disease risk, and especially if opposite of trait effects in adulthood, e.g., if higher IGF axis activity improved cognitive development, but also conferred risk of sharper cognitive decline in adulthood (or vice versa).

The present results, along with inconsistency across previous conventional epidemiologic studies, suggest that any interventions aimed at increasing circulating IGF1/IGFBP3 to reduce $\mathrm{AD}$ risk may fail, and that these traits could be deselected as preventive $\mathrm{AD}$ candidates. The findings do not exclude a role of circulating IGF2 or other growth factors in $\mathrm{AD}$ development or progression, or of IGFs in other forms of neurodegeneration, and future research could investigate these questions further. Gerontologic and genetic studies should also follow up on how FOXO3 and its role in aging may intersect with $\mathrm{AD}$ etiology.

\section{Author contributions}

D.M.W. conceived the study concept, conducted the statistical analysis, and drafted the manuscript. All coauthors were involved in designing the study, interpreting the data, and revising the manuscript for final submission. D.M.W. is guarantor for the research.

\section{Acknowledgment}

The authors thank Alexander Ploner (Department of Medical Epidemiology and Biostatistics, Karolinska Institutet) for advice on statistical modelling of data in this research and Sally Williams for a review of the manuscript prior to acceptance and the International Genomics of Alzheimer's Project (IGAP) for providing summary results data for these analyses. The investigators within IGAP contributed to the design and implementation of IGAP or provided data but did not participate in analysis or writing of this report. IGAP was made possible by the participation of the control subjects, the patients, and their families. The authors also thank the individuals from the Swedish Twin Registry that participated in the cohorts from which genetic data used in this study were collected.

\section{Study funding}

Supported by a European Union Horizon 2020 research and innovation program grant (agreement 634821), the US NIH (grants R01 AG10175, R01 AG08754, R01 AG08861, R01 AG08724, R01 AG028555, and U01 DK066134), the 
MacArthur Foundation Research Network on Successful Aging, the Axel and Margaret Ax:son Johnsons Foundation, the Swedish Research Council (grants 521-2013-8689, 201503255, and 2015-02907), the Göran Gustafsson Foundation, the Swedish Heart-Lung Foundation (grant 20140422), the Knut och Alice Wallenberg Foundation (grant 2013.0126), the Swedish Foundation for Health Care Sciences and Allergy Research, KID (Karolinska Institutet Delfinansiering) grant for doctoral student education (for I.K.K. and N.L.P.), the Loo \& Hans Osterman Foundation, the Foundation for Geriatric Diseases, the Swedish Council for Working Life and Social Research (grant 2013-2292), and The Strategic Research Program in Epidemiology at Karolinska Institutet. For IGAP, the i-Select chips were funded by the French National Foundation on Alzheimer's disease and related disorders. EADI was supported by the LABEX (Laboratory of Excellence Program Investment for the Future) DISTALZ grant, Inserm, Institut Pasteur de Lille, Université de Lille 2, and the Lille University Hospital. GERAD was supported by the Medical Research Council (grant 503480), Alzheimer's Research UK (grant 503176), the Wellcome Trust (grant 082604/2/07/Z), and German Federal Ministry of Education and Research (BMBF): Competence Network Dementia (CND) grant 01GI0102, 01GI0711, 01GI0420. CHARGE was partly supported by NIH/NIA grant R01 AG033193 and NIA AG081220 and AGES contract N01-AG-12100, NHLBI grant R01 HL105756, the Icelandic Heart Association, and the Erasmus Medical Center and Erasmus University. ADGC was supported by NIH/NIA grants U01 AG032984, U24 AG021886, and U01 AG016976, and Alzheimer's Association grant ADGC-10-196728.

\section{Disclosure}

The authors report no disclosures relevant to the manuscript. Go to Neurology.org/N for full disclosures.

Received April 26, 2017. Accepted in final form October 20, 2017.

\section{References}

1. Benarroch EE. Insulin-like growth factors in the brain and their potential clinical implications. Neurology 2012;79:2148-2153.

2. Zadik ZVI, Chalew A, McCarter JRJ, Meistas M, Kowarski AA. The influence of age on the 24-hour integrated concentration of growth hormone in normal individuals. J Clin Endocrinol Metab 1985;60:513-516.

3. Dudek H, Datta SR, Franke TF, et al. Regulation of neuronal survival by the serinethreonine protein kinase akt. Science 1997;275:661-665.

4. Hong M, Lee VMY. Insulin and insulin-like growth factor-1 regulate tau phosphorylation in cultured human neurons. J Biol Chem 1997;272:19547-19553.

5. Dik MG, Pluijm SMF, Jonker C, Deeg DJH, Lomecky MZ, Lips P. Insulin-like growth factor I (IGF-I) and cognitive decline in older persons. Neurobiol Aging 2003;24:573-581.

6. Westwood AJ, Beiser A, DeCarli C, et al. Insulin-like growth factor-1 and risk of Alzheimer dementia and brain atrophy. Neurology 2014;82:1613-1619.

7. Hertze J, Nägga K, Minthon L, Hansson O. Changes in cerebrospinal fluid and blood plasma levels of IGF-II and its binding proteins in Alzheimer's disease: an observational study. BMC Neurol 2014;14:64.

8. Duron E, Funalot B, Brunel N, et al. Insulin-like growth factor-I and insulin-like growth factor binding protein-3 in Alzheimer's disease. J Clin Endocrinol Metab 2012;97:4673-4681.

9. Kalmijn S, Janssen JAMJL, Pols HAP, Lamberts SWJ, Breteler MMB. A prospective study on circulating insulin-like growth factor I (IGF-I), IGF-binding proteins, and cognitive function in the elderly. J Clin Endocrinol Metab 2000;85:4551-4555.

10. Green CJ, Holly JM, Bayer A, et al. The role of IGF-I, IGF-II, and IGFBP-3 in male cognitive aging and dementia risk: the Caerphilly Prospective Study. J Alzheimers Dis 2014;41:867-875.
11. Vardy ERLC, Rice PJ, Bowie PCW, Holmes JD, Grant PJ, Hooper NM. Increased circulating insulin-like growth factor-1 in late-onset Alzheimer's disease. J Alzheimers Dis 2007; 12:285-290.

12. Johansson P, Åberg D, Johansson JO, et al. Serum but not cerebrospinal fluid levels of insulin-like growth factor-I (IGF-I) and IGF-binding protein-3 (IGFBP-3) are increased in Alzheimer's disease. Psychoneuroendocrinology 2013;38:1729-1737.

13. Lawlor DA, Harbord RM, Sterne JA, Timpson N, Davey Smith G. Mendelian randomization: using genes as instruments for making causal inferences in epidemiology. Stat Med 2008;27:1133-1163.

14. Pierce BL, Burgess S. Efficient design for mendelian randomization studies: subsample and 2-sample instrumental variable estimators. Am J Epidemiol 2013;178: $1177-1184$

15. Teumer A, Qi Q, Nethander M, et al. Genomewide meta-analysis identifies loci associated with IGF-I and IGFBP-3 levels with impact on age-related traits. Aging Cell 2016;15:811-824.

16. Kaplan RC, Petersen $\mathrm{AK}$, Chen $\mathrm{MH}$, et al. A genome-wide association study identifies novel loci associated with circulating IGF-I and IGFBP-3. Hum Mol Genet 2011;20: $1241-1251$.

17. Lambert JC, Ibrahim-Verbaas CA, Harold D, et al. Meta-analysis of 74,046 individuals identifies 11 new susceptibility loci for Alzheimer's disease. Nat Genet 2013;45: 1452-1458.

18. Magnusson PK, Almqvist C, Rahman I, et al. The Swedish Twin Registry: establishment of a biobank and other recent developments. Twin Res Hum Genet 2013;16: 317-329.

19. Gatz M, Fratiglioni L, Johansson B, et al. Complete ascertainment of dementia in the Swedish Twin Registry: the HARMONY study. Neurobiol Aging 2005;26: 439-447.

20. Finkel D, Pedersen NL. Processing speed and longitudinal trajectories of change for cognitive abilities: The Swedish Adoption/Twin Study of Aging. Aging Neuropsychol Cogn 2004;11:325-345.

21. Gold CH, Malmberg B, McClearn GE, Pedersen NL, Berg S. Gender and health: a study of older unlike-sex twins. J Gerontol B Psychol Sci Soc Sci 2002;57: S168-S176.

22. Lawlor DA. Commentary: On Gao C, et al. Mendelian randomization study of adiposity-related traits and risk of breast, ovarian, prostate, lung and colorectal cancer. Int J Epidemiol 2016;45:908-915.

23. Burgess S, Small DS, Thompson SG. A review of instrumental variable estimators for mendelian randomization. Stat Methods Med Res 2017;26:2333-2355.

24. Firth SM, Baxter RC. Cellular actions of the insulin-like growth factor binding proteins. Endocr Rev 2002;23:824-854.

25. Juul A, Main K, Blum WF, Lindholm J, Ranke MB, Skakkebaek NE. The ratio between serum levels of insulin-like growth factor (IGF)-I and the IGF binding proteins (IGFBP-1, 2 and 3) decreases with age in healthy adults and is increased in acromegalic patients. Clin Endocrinol 1994;41:85-93.

26. Rich SS, Wang ZY, Sturcke A, et al. Rapid evaluation of phenotypes, SNPs and results through the dbGaP CHARGE summary results site. Nat Genet 2016;48:702-703.

27. Wan X, Wang W, Liu J, Tong T. Estimating the sample mean and standard deviation from the sample size, median, range and/or interquartile range. BMC Med Res Methodol 2014;14:135.

28. Bowden J, Smith GD, Burgess S. Mendelian randomization with invalid instruments: effect estimation and bias detection through Egger regression. Int J Epidemiol 2015; 44:512-525.

29. Burgess S. Sample size and power calculations in mendelian randomization with a single instrumental variable and a binary outcome. Int J Epidemiol 2014;43: 922-929.

30. Hemani G, Zheng J, Wade KH, et al. MR-Base: a platform for systematic causal inference across the phenome using billions of genetic associations. bioRxiv Epub 2016 Dec 16.

31. Salehi Z, Mashayekhi F, Naji M. Insulin like growth factor-1 and insulin like growth factor binding proteins in the cerebrospinal fluid and serum from patients with Alzheimer's disease. BioFactors 2008;33:99-106.

32. Murialdo G, Barreca A, Nobili F, et al. Relationships between cortisol, dehydroepiandrosterone sulphate and insulin-like growth factor-I system in dementia. J Endocrinol Invest 2001;24:139-146.

33. Martins R, Lithgow GJ, Link W. Long live FOXO: unraveling the role of FOXO proteins in aging and longevity. Aging Cell 2015;15:196-207.

34. Broer L, Buchman AS, Deelen J, et al. GWAS of longevity in CHARGE consortium confirms APOE and FOXO3 candidacy. J Gerontol A Biol Sci Med Sci 2015;70: 110-118.

35. Shi C, Viccaro K, Lee HG, Shah K. Cdk5-Foxo3 axis: initially neuroprotective, eventually neurodegenerative in Alzheimer's disease models. J Cell Sci 2016;129: 1815-1830.

36. Tindale LC, Leach S, Spinelli JJ, Brooks-Wilson AR. Lipid and Alzheimer's disease genes associated with healthy aging and longevity in healthy oldest-old. Oncotarget 2017;8:20612-20621.

37. Bonilla C, Lewis SJ, Rowlands MA, et al. Assessing the role of insulin-like growth factors and binding proteins in prostate cancer using mendelian randomization: genetic variants as instruments for circulating levels. Int J Cancer 2016;139:1520-1533.

38. Østergaard SD, Mukherjee S, Sharp SJ, et al. Associations between potentially modifiable risk factors and Alzheimer disease: a mendelian randomization study. PLOS Med 2015;12:e1001841. 


\title{
Circulating insulin-like growth factors and Alzheimer disease
}

\author{
A mendelian randomization study
}

Dylan M. Williams, PhD, Ida K. Karlsson, MSc, Nancy L. Pedersen, PhD, and Sara Hägg, PhD

Correspondence

Cite as: Neurology ${ }^{\otimes}$ 2018;90:e291-e297. doi:10.1212/WNL.0000000000004854

Dr. Williams

dylan.williams@ki.se

\section{Study question}

Are genetically predicted variations in circulating insulin-like growth factor 1 (IGF1) or its binding protein, IGFBP3, associated with the risk of developing Alzheimer disease ( $\mathrm{AD})$ ?

\section{Summary answer}

Circulating IGF1 and IGFBP3 were not important determinants of the risk of developing $\mathrm{AD}$.

\section{What is known and what this article adds}

Epidemiologic evidence suggests that circulating IGF1 and IGFBP3 levels are lower in individuals who develop $\mathrm{AD}$ than in cognitively intact individuals. However, this study provides evidence against the hypothesis that circulating IGF1 levels are a modifiable target in $\mathrm{AD}$ treatments.

\section{Design, size, and duration}

This study involved mendelian randomization with 2 steps. In step 1 , genetic variants that affect circulating IGF1 and IGFBP3 levels were identified in published genetic datasets. In step 2, genotype-outcome associations for identified genetic variants were assessed in $\mathrm{AD}$ case-control datasets.

\section{Participants and setting}

The step 1 analyses for IGF1 drew on data obtained from 30,884 individuals from 21 cohort studies. Those for IGFBP3 drew on data for 18,995 individuals from 13 cohort studies. The step 2 analyses relied on data for 17,008 patients with late-onset $\mathrm{AD}$ and 37,154 controls. A secondary case-control dataset included 984 patients and 10,304 controls. All participants were of European ancestry.

\section{Primary outcomes}

The primary outcome for these analyses was the development of $\mathrm{AD}$.

\section{Main results and the role of chance}

Genetically determined, long-term exposure to IGF1 and IGFBP3 variation did not affect the risk of developing $\mathrm{AD}$. The only individual genotype that increased the risk of developing $\mathrm{AD}$ was rs2153960, which lies within the FOXO3 gene. The odds ratio of $\mathrm{AD}$ per $\mathrm{FOXO3}$ risk allele was 1.04 (95\% confidence interval 1.01-1.08; $p=0.008$ ).

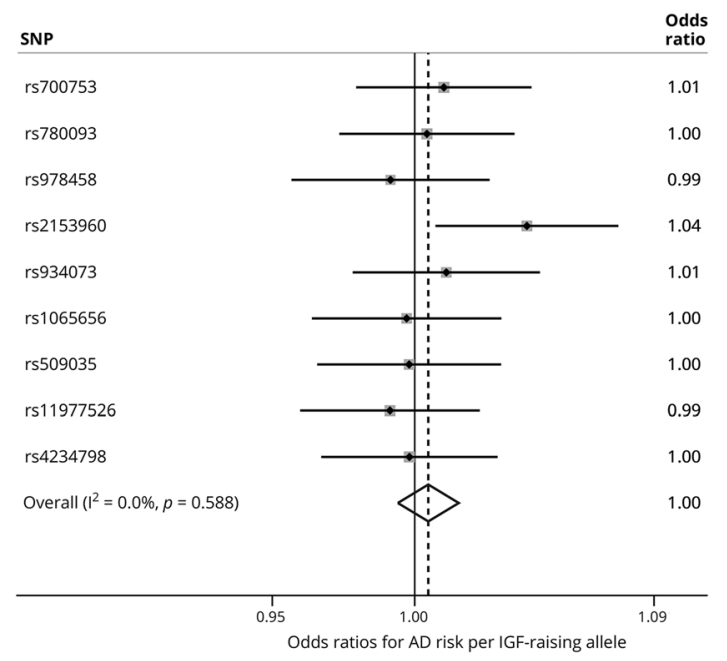

\section{Bias, confounding, and other reasons} for caution

The datasets for steps 1 and 2 may have partially overlapped, but the effect on results would be negligible. The analyzed variants may not exclusively affect IGF1 or IGFBP3, but provide a proxy for exposure to overall IGF axis activity on disease risk. The study also assesses the lifelong averaged effect of IGFs on $\mathrm{AD}$ risk, which could mask any antagonistic effects of exposure on disease risk at different life stages. While mendelian randomization designs are expected to be robust to confounding and reverse causation, the prospect of bias from genetic pleiotropy cannot be completely excluded with current methods.

\section{Generalizability to other populations}

The results may not be generalizable to populations of nonEuropean ancestry.

\section{Study funding/potential competing interests}

This study was funded by various governments and various medical research foundations. The authors report no conflicts of interest. Go to Neurology.org/N for full disclosures. 


\section{Neurology}

\section{Circulating insulin-like growth factors and Alzheimer disease: A mendelian randomization study}

Dylan M. Williams, Ida K. Karlsson, Nancy L. Pedersen, et al.

Neurology 2018;90;e291-e297 Published Online before print December 27, 2017

DOI 10.1212/WNL.0000000000004854

\section{This information is current as of December 27, 2017}

\section{Updated Information \& Services}

References

Subspecialty Collections

Permissions \& Licensing

Reprints including high resolution figures, can be found at: http://n.neurology.org/content/90/4/e291.full

This article cites 37 articles, 5 of which you can access for free at: http://n.neurology.org/content/90/4/e291.full\#ref-list-1

This article, along with others on similar topics, appears in the following collection(s):

Alzheimer's disease

http://n.neurology.org/cgi/collection/alzheimers_disease Association studies in genetics

http://n.neurology.org/cgi/collection/association_studies_in_genetics Risk factors in epidemiology

http://n.neurology.org/cgi/collection/risk_factors_in_epidemiology

Information about reproducing this article in parts (figures,tables) or in its entirety can be found online at:

http://www.neurology.org/about/about_the_journal\#permissions

Information about ordering reprints can be found online:

http://n.neurology.org/subscribers/advertise

Neurology ${ }^{\circledR}$ is the official journal of the American Academy of Neurology. Published continuously since 1951, it is now a weekly with 48 issues per year. Copyright Copyright (C) 2017 The Author(s). Published by Wolters Kluwer Health, Inc. on behalf of the American Academy of Neurology.. All rights reserved. Print ISSN: 0028-3878. Online ISSN: 1526-632X.

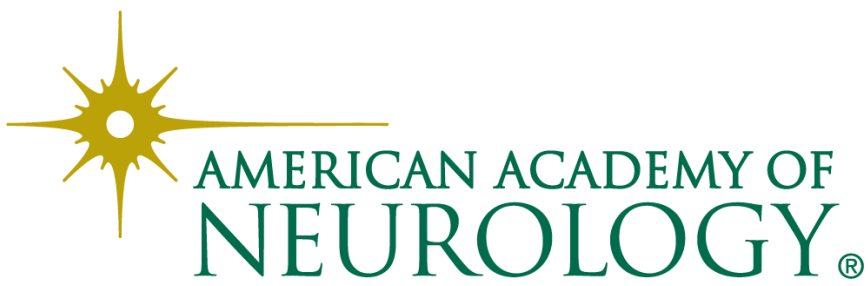

